\title{
Multi-Spectral Large Area Montage of a Heavy Metal Contaminated Butterfly
}

\author{
W.J. Mershon, ${ }^{*}$ J.E. Rawlins, ${ }^{* *}$ G.H. Cooper*** \\ * Tescan USA, 508 Thomson Park Dr, Cranberry Township PA 16066 \\ ** Carnegie Museum of Natural History, Pittsburgh, PA 15213 \\ ***Four Chambers Studio, Vallejo, CA 94589
}

Many hundreds of dry, pinned butterfly specimens in the research collections at Carnegie Museum of Natural History $(\mathrm{CMNH})$ are distinctive in that they are darkened and coated with what seems like a fine black dust. Almost all such blackened specimens were collected between 1870 and 1910, especially specimens purchased from the great European entomological specimen dealer, Otto Staudinger, in Dresden. The specimens represent many species, large and small, and are notably free of damage from insect pests that feed on dry preserved insect specimens (especially larval dermestids). The black material was surmised to be mercury, and this is here confirmed by energy dispersive $\mathrm{x}$-ray analysis. During the 19th and early 20th centuries, aqueous mercuric chloride ( $\mathrm{HgCl} 2$ or "corrosive sublimate") was used to preserve specimens in botany, entomology, and general zoology against museum pests such as dermestids. Specimens were dipped or lightly sprayed with $2 \%$ solutions to prevent mold, fungi, and insect damage. Mercuric chloride was widely used during this period as a killing agent, a fixative, a narcotizing agent, and a long term preservative, with practitioners not fully appreciating the danger from handling it or absorbing it by accident.[1]

This butterfly is just one of several million specimens in the collection of the CMNH. A goal of the museum is to create an automated microscopy system that could collect high spatial resolution montages across an entire specimen to make virtual observation and analysis a possibility. A wingspan of 50000um requires the acquisition of over one billion pixels to provide micron scale sampling across the entire surface of the butterfly. Complete observation is greatly enhanced by combining optical from a light microscope and SE BSE and EDX mapping from an SEM and correlating them on a micron scale. The solution is to collect optical gigapan montage of the entire object and then move to the SEM and collect SE, BSE, montages as well as a montage of EDX full spectrum maps. All of these pixels are then stitched together tile-by-tile and layer-by-layer to create a multi-layer, point-correlated map. That is easily navigated over centimeters in a browser and quickly zoomed to a micron resolution for detailed analysis without the need to collect additional image data from the specimen.

The visible light gigapixel imaging of the butterfly dorsal and ventral sides is accomplished by photographing the subject using a macro linear robotic imaging device called a GigaMacroBot. The GigaMacroBot takes thousands of images of the subject capturing microscopic detail throughout the specimen. By combining focus stacking techniques to increase depth of field and image mosaicing a final seamless image can be produced. The final image is then and uploaded to a website for online exploration and viewing.

The SEM data was collected in a Tescan Vega3 VP SEM equipped with software for automated montage collection and a Bruker Quantax 800 EDX analyzer equipped with a $30 \mathrm{~mm}$ Sq silicon drift detector. All montages were collected using variable pressure operation. The SE data was collected at 2048 X 2048 pixels, 10kV, $30 \mathrm{~Pa}$ chamber pressure, 35mm, 32us/pixel, and $1.92 \mathrm{um} /$ pixel. The 
BSE montage was collected at 2048 X 2048 pixels, 30kV, $30 \mathrm{~Pa}, 35 \mathrm{~mm}, 8 \mathrm{us} /$ pixel and 1.22um/pixel. The EDX maps were collected at 512 X 384 pixels, 30kV, 30Pa, 20mm, 16us/pixel, 8um/pixel at input count rates of $20-100 \mathrm{kcps}$, for a total of 300 seconds of live time.

This project succeeded in correlating the blackening of specimens with the presence of mercury that was deposited as a preserving compound. The project also succeeded in creating four gigapixel panoramas collected in two different instruments in an automated manner and establishing pixel by pixel between them.

The next steps involve quantifying how much mercury is on the butterfly in what form and how it is bound to the surface. There also needs to be an improved technique for mounting the specimen in terms of stability, registration and protection from damage. Integrating EDX collection with the SEM image collection would significantly improve the productivity of the SEM data collection and advance to the goal of virtual observation of collection in the future.

\section{References}

[1] L. Goldberg, JAIC 1996, Volume 35, Number 1, Article 3, pp(23-43).
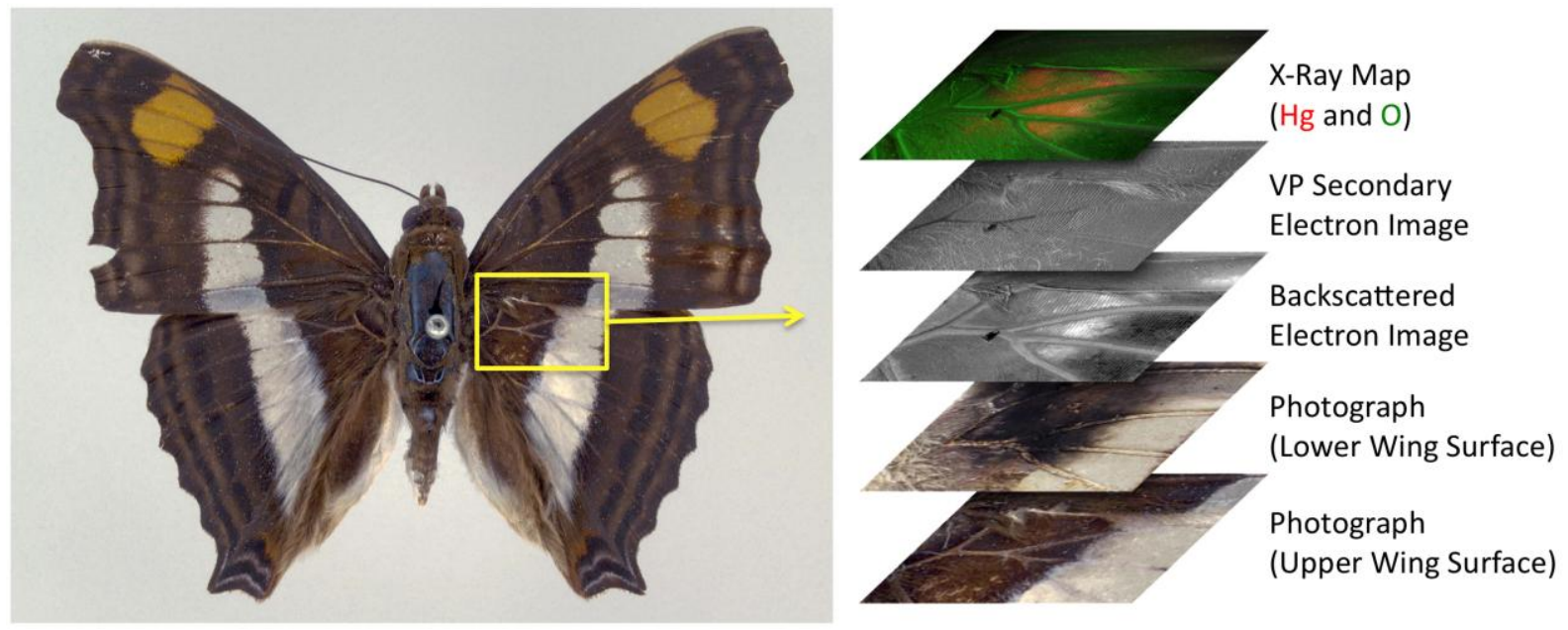

Fig. 1. Illustration of the spatial relationships of the optical, SEM and EDX montage data.

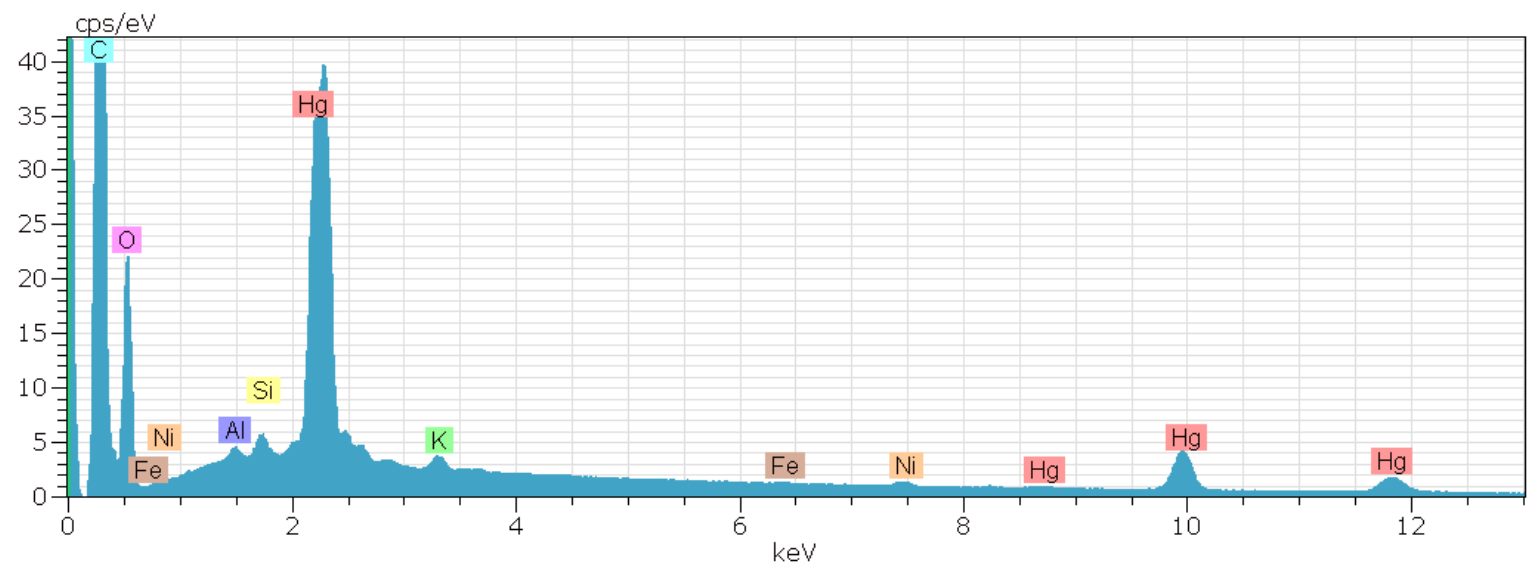

Fig. 2. EDX spectrum extracted from the x-ray map showing a high concentration of mercury. 